\title{
On Three-Point Finite Difference Techniques for SPBVPs
}

\author{
E. R. El-Zahar ${ }^{1,2 *}$ A.M. Algelany ${ }^{1,3}$ \\ ${ }^{1}$ Department of Mathematics, College of Science and Humanities in Al-Kharj, \\ Prince Sattam bin Abdulaziz University, P.O. Box 83, Al-Kharj, 11942, Saudi Arabia. \\ ${ }^{2}$ Department of Basic Engineering Science, Faculty of Engineering, \\ Shebin El-Kom, 32511, Menofia University, Egypt. \\ ${ }^{3}$ Department of Mathematics, Faculty of Sciences, Fayoum University, Fayoum, Egypt.
}

*Corresponding Author: ORCID: 0000-0001-7266-1893(E.R.El-Zahar)

\begin{abstract}
In this paper, three finite difference three-point techniques for singularly perturbed boundary value problems (SPBVPs) are discussed. These techniques are developed over unevenly spaced grid points aided mathematical symbolic language Maple. Local truncation error, uniqueness and stability conditions are discussed.
\end{abstract}

Keywords: Finite difference three-point techniques; unevenly spaced grid; convergence; stability.

\section{INTRODUCTION}

Singularly perturbed boundary value problems (SPBVPs) arise frequently in applied sciences and engineering and have been extensively studied in recent years [1-16]. It is well known that away from the boundary layers, upwind difference methods can be used and accurate results are obtained. Otherwise, other schemes are to be preferred such as difference schemes on a non-uniform mesh [1-6, 8-10]. But in this case one must face the drawbacks related to the use of difference schemes on highly non-uniform meshes, since a fine mesh with maximum step-size $h<\varepsilon$ is required over a domain containing the layer region at which the solution varies rapidly, while for reasons of efficiency a coarse grid with $h \gg \varepsilon$ should be used in the outer region at which the solution behaves regularly and changes slowly. The main difficulty in global discretization of these problems is the restriction on the step- size that to have a unique stable and accurate solution. Therefore stability and order of convergence act as the major achieved requirements. Many authors deal with some of these challenges in global discretization for these problems especially the convection diffusion problems [2-6]. Segal [2] analyzed and compared various methods for solving the convection diffusion equation with small $\varepsilon$. While Il'in's [3] method is a very accurate example of an upwind scheme for a homogeneous, onedimensional convection-diffusion equation with constant coefficients. It loses accuracy when variable coefficients are used. Dekema and Schultz [4] developed high-order methods to solve elliptic singular perturbation problems and obtained remarkably good numerical results. Later, Choo and Schultz [5] developed the so-called stable central difference methods. They modified the central difference approximations for the first- and second-order derivatives by rewriting its error terms as a combination of the lower-order derivative terms and approximating them. This process reinforced the diagonal dominance of the coefficient matrix and had a stabilizing effect. However, they could not achieve as high accuracy as the method of Dekema and Schultz. Ilicasu and Schultz [6] developed high-order methods to solve singular perturbation problems. They rewrote higher order derivatives in Taylor expansion in terms of the lower-order derivative terms. However, they also used constant coefficients only. Most the above techniques go a way from using non-uniform grid points. The main reason is the complexity of driving general formulas that will solve these problems. Moreover, this leads to more complicated studying of uniqueness, stability, and convergence. Now, using mathematical symbolic language such as Maple, Drive and Matlab makes the mission easier than earlier. In this paper, following the idea in [6] three finite difference three-point techniques for singularly perturbed boundary value problems (SPBVPs) are suggested. These techniques are developed over unevenly spaced grid points aided mathematical symbolic language Maple. Local truncation error, uniqueness and stability conditions are discussed

\section{FINITE DIFFERENCE TECHNIQUES}

Consider the following linear SPBVP

$$
-\varepsilon y^{\prime \prime}+p(x) y^{\prime}+q(x) y=f(x), \quad a \leq x \leq b,
$$

with boundary conditions

$$
y(a)=\alpha \text { and } y(b)=\beta,
$$

where $\varepsilon$ is a small positive parameter $(0<\varepsilon \ll 1), \quad \alpha$ and $\beta$ are given constants, $p(x), q(x)$ and $f(x)$ are assumed to be sufficiently continuously differentiable functions on $[a, b]$, Moreover assume $q(x)>0, p(x)<P<0$ for all $x \in[a, b]$, where $P$ is some negative constant. Under these assumptions, SPBVP (1) has a unique solution which in general displays a boundary layer of width $O(\varepsilon)$ at $x=a[2,4,6-16]$. First, $[a, b]$ is divided into $N$ non-equal subintervals such that $\pi: x_{0}=a<x_{1}<x_{2}<\ldots . .<x_{N}=b \quad$ with $\quad h_{i}=x_{i}-x_{i-1}$, 
$i=1,2, \ldots . . N$. For the sake of simplicity, we will use $p_{i}=p\left(x_{i}\right), q_{i}=q\left(x_{i}\right), f_{i}=f\left(x_{i}\right), y_{i-1}=y\left(x_{i-1}\right)$,

$y_{i+1}=y\left(x_{i+1}\right)$, and $y_{i}^{\prime}=y^{\prime}\left(x_{i}\right)$, etc.

The solution of SPBVP (1) is approximated over subintervals with unevenly spaces three grid points as shown in figure 1 .

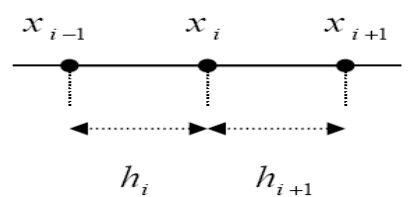

Figure 1. Unevenly spaces grid points over sub-domains

Equation (1) is divided by $-\varepsilon$ and we let $\omega=1 / \varepsilon$. At each $x_{i}$, we want to find $E_{i}, F_{i}, G_{i}, H_{i}$ such that

$$
y_{i}^{\prime \prime}-\omega p_{i} y_{i}^{\prime}-\omega q_{i} y_{i}=E_{i} y_{i-1}+F_{i} y_{i}+G_{i} y_{i+1}+H_{i}=-\omega f_{i}
$$

These terms are obtained using Taylor series expansions of $y_{i+1}$ and $y_{i-1}$ around $x_{i}$

$$
\begin{array}{r}
y_{i}^{\prime \prime}-\omega p_{i} y_{i}^{\prime}-\omega q_{i} y_{i}=F_{i} y_{i}+H_{i}+G_{i}\left[y_{i}+h_{i} y_{i}^{\prime}+\frac{h_{i+1}^{2}}{2} y_{i}^{\prime \prime}\right. \\
\left.+\frac{h_{i+1}^{3}}{6} y_{i}^{\prime \prime \prime}+. .\right]+E_{i}\left[y_{i}-h_{i} y_{i}^{\prime}+\frac{h_{i}^{2}}{2} y_{i}^{\prime \prime}-\frac{h_{i}^{3}}{6} y_{i}^{\prime \prime \prime}+. .\right]
\end{array}
$$

From Eq. (1) we have

$$
\begin{aligned}
y_{i}^{\prime \prime \prime}= & \omega p_{i} y_{i}^{\prime \prime}+\omega\left(p_{i}^{\prime}+q_{i}\right) y_{i}^{\prime}+\omega q_{i}^{\prime} y_{i}-\omega f_{i}^{\prime} \\
y_{i}^{(4)} & =\left[\omega^{2} p_{i}^{2}+2 \omega p_{i}^{\prime}+\omega q_{i}\right] y_{i}^{\prime \prime}-\omega f_{i}^{\prime \prime}+\left[\omega^{2} p_{i}\left(p_{i}^{\prime}+q_{i}\right)+\right. \\
& \left.+\omega\left(p_{i}^{\prime \prime}+2 q_{i}^{\prime}\right)\right] y_{i}^{\prime}+\left[\omega^{2} p_{i} q_{i}^{\prime}+\omega q_{i}^{\prime \prime}\right] y_{i}-\omega^{2} p_{i} f_{i}^{\prime} \\
y_{i}^{(5)} & =\left[\omega^{3} p_{i}^{3}+5 \omega^{2} p_{i}^{\prime} p_{i}+3 \omega p_{i}^{\prime \prime}+3 \omega q_{i}^{\prime}+2 \omega^{2} p_{i} q_{i}\right] y_{i}^{\prime \prime} \\
& +\left[\omega^{3} p_{i}^{2} p_{i}^{\prime}+\omega^{3} p_{i}^{2} q_{i}+3 \omega^{2} p_{i}^{\prime 2}+4 \omega^{2} p_{i}^{\prime} q_{i}+3 \omega q_{i}^{\prime \prime}+\right. \\
& \left.+2 \omega^{2} p_{i} q_{i}^{\prime}+\omega^{2} p_{i} p_{i}^{\prime \prime}+\omega^{2} q_{i}^{2}+\omega p_{i}^{\prime \prime \prime}\right] y_{i}^{\prime}+\left[\omega^{3} p_{i}^{2} q_{i}^{\prime}+\right. \\
& \left.+3 \omega^{2} p_{i}^{\prime} q_{i}^{\prime}+\omega^{2} q_{i} q_{i}^{\prime}+\omega^{2} p_{i} q_{i}^{\prime \prime}+\omega q_{i}^{\prime \prime \prime}\right] y_{i}- \\
& -\left[\omega^{3} p_{i}^{2} f_{i}^{\prime}+3 \omega^{2} p_{i}^{\prime} f_{i}^{\prime}+\omega^{2} q_{i} f_{i}^{\prime}+\omega^{2} p_{i} f_{i}^{\prime \prime}+\omega f_{i}^{\prime \prime \prime}\right] .
\end{aligned}
$$

\section{II.I. Technique-I}

Substituting Eq.(4) in Eq.(3) and equating the coefficients of $y_{i}, y_{i}^{\prime}$ and $y_{i}^{\prime \prime}$, taking the third order derivative terms are the largest contributors to the error, we get

$$
\begin{aligned}
& E_{i}=\frac{2+h_{i+1} \omega p_{i}}{h_{i}\left(h_{i}+h_{i+1}\right)}, F_{i}=-G_{i}-E_{i}-\omega q_{i}, \\
& G_{i}=\frac{2-h_{i} \omega p_{i}}{h_{i+1}\left(h_{i}+h_{i+1}\right)}, H_{i}=0
\end{aligned}
$$

Then, the difference equation of technique $\mathrm{I}$ and local truncation error $\tau_{i}$ are introduced as

$$
\begin{aligned}
& E_{i} y_{i-1}+F_{i} y_{i}+G_{i} y_{i+1}=-H_{i}-w f_{i}+\tau_{i}, \\
\tau_{i}= & \left(G_{i} \frac{h_{i+1}^{3}}{6} y^{(3)}(\xi)-E_{i} \frac{h_{i}^{3}}{6} y^{(3)}(\zeta)\right),
\end{aligned}
$$

where $\xi \in\left[x_{i}, x_{i+1}\right], \zeta \in\left[x_{i-1}, x_{i}\right]$.

\section{II.II. Technique-II}

Substituting Eq. (4) in Eq. (3) and equating the coefficients of $y_{i}, y_{i}^{\prime}$ and $y_{i}^{\prime \prime}$, taking the fourth order derivative terms are the largest contributors to the error, we get

$$
\left.\begin{array}{l}
E_{i}=\frac{2\left(h_{i+1}^{2} \omega p_{i}^{\prime}+h_{i+1}^{2} \omega q_{i}+3 h_{i+1} \omega p_{i}+h_{i+1}^{2} \omega^{2} p_{i}^{2}+6\right)}{h_{i} T} \\
G_{i}=\frac{2\left(h_{i}^{2} \omega p_{i}^{\prime}+h_{i}^{2} \omega q_{i}-3 h_{i} \omega p_{i}+h_{i}^{2} \omega^{2} p_{i}^{2}+6\right)}{h_{i+1} T} \\
F_{i}=-G_{i}\left(1+h_{i+1}^{3} q_{i}^{\prime} \omega / 6\right)-E_{i}\left(1-h_{i}^{3} q_{i}^{\prime} \omega / 6\right)-\omega q_{i} \\
H_{i}=G_{i}\left(h_{i+1}^{3} f_{i}^{\prime} \omega / 6\right)-E_{i}\left(h_{i}^{3} f_{i}^{\prime} \omega / 6\right)
\end{array}\right\},
$$

where $T=\left(h_{i}+h_{i+1}\right)\left[h_{i+1} h_{i} \omega\left(p_{i}^{\prime}+q_{i}\right)+2 p_{i} \omega\left(h_{i+1}-h_{i}\right)+6\right]$.

Then, the difference equation of technique II and local truncation error $\tau_{i}$ are introduced as

$$
\begin{aligned}
& E_{i} y_{i-1}+F_{i} y_{i}+G_{i} y_{i+1}=-H_{i}-w f_{i}+\tau_{i}, \\
& \tau_{i}=\left(G_{i} \frac{h_{i+1}^{4}}{24} y^{(4)}(\xi)+E_{i} \frac{h_{i}^{4}}{24} y^{(4)}(\zeta)\right),
\end{aligned}
$$

where $\xi \in\left[x_{i}, x_{i+1}\right], \zeta \in\left[x_{i-1}, x_{i}\right]$.

\section{II.III. Technique-III}

Substituting Eq.(4) in Eq.(3) and equating the coefficients of $y_{i}, y_{i}^{\prime}$ and $y_{i}^{\prime \prime}$, taking the fifth order derivative terms are the largest contributors to the error, we get

$$
\left.\begin{array}{rl}
E_{i}= & \frac{6\left(h_{i+1}^{3} \omega \theta_{1}+4 h_{i+1}^{2} \omega\left(p_{i}^{\prime}+p_{i}^{2} \omega+q_{i}\right)+12 h_{i+1} p_{i} \omega+24\right)}{h_{i} T} \\
G_{i}= & \frac{6\left(-h_{i}^{3} \omega \theta_{1}+4 h_{i}^{2} \omega\left(p_{i}^{\prime}+p_{i}^{2} \omega+q_{i}\right)-12 h_{i} p_{i} \omega+24\right)}{h_{i+1} T} \\
F_{i}= & -G_{i}\left[1+\frac{h_{i+1}^{4} \omega \theta_{2}}{24}+\frac{h_{i+1}^{3} q_{i}^{\prime} \omega}{6}\right]-E_{i}\left[1+\frac{h_{i}^{4} \omega \theta_{2}}{24}-\right. \\
& \left.-\frac{\left.h_{i}^{3} q_{i}^{\prime} \omega\right]-\omega q_{i}}{6}\right], \\
H_{i}= & G_{i}\left[\frac{h_{i+1}^{4} \omega \theta_{3}}{24}+\frac{h_{i+1}^{3} f_{i}^{\prime} \omega}{6}\right]+E_{i}\left[\frac{h_{i}^{4} \omega \theta_{3}}{24}-\frac{h_{i}^{4} f_{i}^{\prime} \omega}{6}\right]
\end{array}\right\}
$$


where

$$
\begin{aligned}
\theta_{1}= & p_{i}^{\prime \prime}+2 q_{i}^{\prime}+p_{i} \omega\left(3 p_{i}^{\prime}+p_{i}^{2} \omega+2 q_{i}\right) \\
\theta_{2}= & q_{i}^{\prime} p_{i} \omega+q_{i}^{\prime \prime} \\
\theta_{3}= & \omega\left(f_{i}^{\prime} p_{i} \omega+f_{i}^{\prime \prime}\right) \\
T= & 6 \omega\left(h_{i}^{3}+h_{i+1}^{3}\right)\left(2 p_{i}^{\prime}+p_{i}^{2} \omega+q_{i}\right)+3 \omega\left(h_{i+1}^{2}-h_{i}^{2}\right)\left(8 p_{i}+\right. \\
+ & \left.h_{i} h_{i+1}\left(2 q_{i}^{\prime}+p_{i}^{\prime} p_{i} \omega+p_{i}^{\prime \prime}+p_{i} q_{i} \omega\right)\right)-\left(h_{i+1}+h_{i}\right) \\
& \left(\omega^{2} h_{i}^{2} h_{i+1}^{2}\left(2 q_{i}^{\prime} p_{i}-2 p_{i}^{\prime 2}-3 p_{i}^{\prime} q_{i}+p_{i}^{\prime \prime} p_{i}-q_{i}^{2}\right)+\right. \\
+ & \left.12 \omega h_{i} h_{i+1}\left(p_{i}^{\prime}+q_{i}\right)+72\right)
\end{aligned}
$$

Then, the difference equation of technique III and local truncation error $\tau_{i}$ are introduced as

$$
\begin{aligned}
& E_{i} y_{i-1}+F_{i} y_{i}+G_{i} y_{i+1}=-H_{i}-\omega f_{i}+\tau_{i}, \\
& \tau_{i}=\left(G_{i} \frac{h_{i+1}^{5}}{120} y^{(5)}(\xi)-E_{i} \frac{h_{i}^{5}}{120} y^{(5)}(\zeta)\right)
\end{aligned}
$$

where $\xi \in\left[x_{i}, x_{i+1}\right], \zeta \in\left[x_{i-1}, x_{i}\right]$.

\section{UNIQUENESS AND STABILITY ANALYSIS}

The existence and uniqueness of the solution for the difference techniques defined in section II is shown by establishing that the tridiagonal coefficient matrix of the result algebraic system is diagonally dominant with negative main diagonal elements and positive super-diagonal and subdiagonal elements.

\section{I. Technique I.}

It clear that $E_{i}$ and $G_{i}$ in (5) are positive under the condition

$$
h_{i}, h_{i+1}<\frac{2}{\omega\left|p_{i}\right|} \text {. }
$$

And since $q>0$, we have

$$
F_{i}=-\left(G_{i}+E_{i}+\omega q_{i}\right)<0
$$

and

$$
\left|F_{i}\right|=\left|G_{i}+E_{i}+\omega q_{i}\right| \geq\left|G_{i}+E_{i}\right|
$$

Thus the numerical technique $\mathrm{I}$ is stable and has a unique solution under condition (14).

\section{II. Technique II.}

It can be easily shown from (8) with constant coefficient $q$, that the nominators of $E_{i}$ and $G_{i}$ are positive with no restrictions on the step size while the denominator $T$ is positive when

$$
h_{i+1} h_{i} \omega\left(p_{i}^{\prime}+q\right)+2 p_{i} \omega\left(h_{i+1}-h_{i}\right)+6>0
$$

thus

$$
h_{i+1}>\left(h_{i}-\frac{3}{\omega p_{i}}-\frac{\left(q_{i}+p_{i}^{\prime}\right) h_{i} h_{i+1}}{2 p_{i}}\right) \text {, }
$$

Thus the numerical technique II is stable and has a unique solution under condition (17).

\section{III. Technique III.}

The nominators of $E_{i}$ and $G_{i}$, in (11) with constant coefficients $p$ and $q$, are positive when $12 h_{i+1} p \omega \leq 24$ and $h_{i+1}^{3} \omega\left(p \omega\left(p^{2} \omega+2 q\right)\right)-4 h_{i+1}^{2} \omega\left(p^{2} \omega+q\right) \leq 0$. Now, let $h_{i+1}=\frac{k}{\omega p}$, then the first condition yields $k \leq 2$, and the second condition yields

$$
\begin{aligned}
& h_{i+1}^{3} \omega\left(p \omega\left(p^{2} \omega+2 q\right)\right)-4 h_{i+1}^{2} \omega\left(p^{2} \omega+q\right) \leq 0 \\
& h_{i}\left(p \omega\left(p^{2} \omega+2 q\right)\right) \leq 4\left(p^{2} \omega+q\right) \\
& \left.k\left(p^{2} \omega+2 q\right)\right) \leq 4\left(p^{2} \omega+q\right)
\end{aligned},
$$

Thus

$$
k \leq \frac{4\left(p^{2} \omega+q\right)}{\left.\left(p^{2} \omega+2 q\right)\right)}, \text { or } k \leq 2,
$$

The denominator will be

$$
\begin{array}{r}
\left.T=6 \omega\left(h_{i}^{3}+h_{i+1}^{3}\right)\left(p^{2} \omega+q\right)+3 \omega\left(h_{i+1}^{2}-h_{i}^{2}\right)\left(8 p+h_{i} h_{i+1} p q \omega\right)\right) \\
+\left(h_{i+1}+h_{i}\right)\left(\omega^{2} h_{i} h_{i+1}^{2} q^{2}+12 \omega h_{i} h_{i+1} q+72\right)
\end{array}
$$

If we substitute by $h_{i}=\frac{L}{\omega p}$ and $h_{i+1}=\frac{M}{\omega p}$ in the denominator:

$$
\left.T=6 \omega\left(h_{i}^{3}+h_{i+1}^{3}\right)\left(p^{2} \omega+q\right)+3 \omega\left(h_{i+1}^{2}-h_{i}^{2}\right)\left(8 p+h_{i} h_{i+1} p q \omega\right)\right)
$$$$
+\left(h_{i+1}+h_{i}\right)\left(\omega^{2} h_{i} h_{i+1}^{2} q^{2}+12 \omega h_{i} h_{i+1} q+72\right)
$$

we get $M \leq 2$, or $M \geq 3$, and $L \leq 3$, or $L \geq 4$, which means that there is no restrictions on the step size obtained from the denominator. Thus the numerical scheme is stable and has a unique solution under the condition (20).

$$
h_{i} ; h_{i+1} \leq \frac{2}{\omega|p|} \text {. }
$$

\section{CONCLUSION AND DISCUSSION}

In this paper, we have presented three finite difference threepoint techniques for singularly perturbed boundary value problems (SPBVPs). These techniques are developed over unevenly spaced grid points aided mathematical symbolic language Maple. Local truncation error, uniqueness and stability conditions are discussed. The paper draws the attention of researchers to drive general formulas over arbitrary grid points and perform deeply more complicated studying of uniqueness, stability, and convergence aided mathematical symbolic language.

Acknowledgement. This Publication was supported by the Deanship of Scientific Research at Prince Sattam bin Abdulaziz University, Saudi Arabia. 


\section{REFERENCES}

[1] Ascher, U Mattheij, RMM. Russel, R.D. Numerical Solution of Boundary Value Problems for ordinary Differential Equations (Prentice-Hall, Englewood Cliffs, N J, 1988).

[2] Segal, A. Aspects of numerical methods for elliptic singular perturbation problems, SIAM J. Sci. Stat.Comput. 3(1982) 327-349.

[3] Dekema, SK., Schultz, DH. High-order methods for differential equations with large first-derivative terms, Int. J. Num. Methods Fluids 10, 259-284, (1990).

[4] Choo, JY. Schultz, DH. Stable high order methods for differential equations with small coefficients for the second order terms, Computers Math. Applic, 25 (1993)105-123.

[5] Il'in, AM. Differencing scheme for a differential equation with a small parameter affecting the highest derivative, Math. Notes Acad. SC. USSR 6, (1969) 596-602.

[6] ILICASU, F0. SCHULTZ, D.H. High-Order FiniteDifference Techniques for Linear Singular Perturbation Boundary Value Problems, Computers Math. Applic, 47 (2004) 391-417

[7] R.Vulanovic, Novi Sad, A uniform numerical method for quasilinear singular perturbation problems without turning points, Computing 41, 97-106(1989).

[8] Alessandra Papini, About the central difference method for singularly perturbed boundary value problems, Appl. Num. Math, 17 (1995) 333-346

[9] Weiss, R. An analysis of the box and trapezoidal schemes for linear singularly perturbed boundary value problems, Math. Comp, 42 (1984)41-67.

[10] Varner, TN. and Choudhury, S.R. Nonstandard difference schemes for singular perturbation problems revisited, Appl. Math. Comp, 92, 101-123, (1998).

[11] Habib HM, El-Zahar ER. An algorithm for solving singular perturbation problems with mechanization. Appl Math Comput, 2007, 188:286-302.

[12] El-Zahar, ER. Piecewise approximate analytical solutions of high order singular perturbation problems with a discontinuous source term, International Journal of Differential Equations, vol. 2016, Article ID 1015634, 12 pages, 2016. doi:10.1155/2016/1015634

[13] El-Zahar, ER. Applications of Adaptive Multi step Differential Transform Method to Singular Perturbation Problems Arising in Science and Engineering, Applied Mathematics and Information Sciences, 2015, 9(1), 223-232.

[14] El-Zahar, ER. Approximate analytical solutions of singularly perturbed fourth order boundary value problems using differential transform method, Journal of King Saud University (Science), 2013, 25(3), 257265.
[15] El-Zahar, ER, EL-Kabeir, SM. A new method for solving singularly perturbed boundary value problems, Applied Mathematics and Information Sciences., 2013, 7(3), 927-938.

[16] El-Zahar, ER. Cubic Spline Solution of Nonlinear Singularly Perturbed Boundary Value Problems via Initial Value Method, International Journal of Engineering Research and Technology, 2019, 12(12), 3145-3150. 